\title{
L'innovation dans l'Aérospatial et la Défense : contribution scientifique et qualité des brevets
}

\author{
Aerospace and Defense innovation: scientific knowledge and patent \\ quality
}

\author{
Cécile Fauconnet ${ }^{1}$ \\ ${ }^{1}$ Laboratoire Unité d'économie appliquée (UEA), ENSTA ParisTech, Université Paris 1 Panthéon-Sorbonne, France, \\ cecile.fauconnet@ensta-paristech.fr
}

\begin{abstract}
RÉSUMÉ. Cet article a pour objet d'étudier l'évolution du modèle d'innovation dans le secteur de l'Aérospatial et de la Défense (A\&D) entre 1945 et 2015. Dans cet article, nous proposons d'évaluer les évolutions du modèle d'innovation du secteur de l'Aérospatial et la Défense (A\&D ci-après) à l'aune de cette vision de l'économie. Nous défendons l'idée que le secteur de I'A\&D ne fait pas figure d'exception face à cette évolution des modèles d'innovation et plus précisément en termes d'utilisation des connaissances scientifiques. Afin de répondre à cette question, nous menons une étude empirique des données de brevets. Cette analyse met en évidence l'augmentation constante des citations de littérature scientifique dans les brevets du secteur depuis les années 1980. Nous montrons aussi une nette évolution dans les pratiques d'innovation de ce secteur qui intègrent de plus en plus des savoirs divers, en particulier issus de la recherche scientifique.

ABSTRACT. This paper looks more closely at the evolution of innovation models in Aerospace and Defense (A\&D) which took place over a long time period (1945-2015). Our main goal is to show the emergence and development of knowledge based-economy through an analysis of innovation models. To do that, we develop an empirical analysis of patents in technological fields of A\&D. This study points at a constant growth of non-patent literature in patent citations since 1980. In order to deepen this analysis, we study the correlation between non patent literature citation and patent quality in A\&D technological fields and we find a clear development in innovative practices, which include more and more diversified knowledge, in particularly scientific knowledge.

MOTS-CLÉS. Innovation, Science et Technologie, Brevet, Aérospatial et Défense.

KEYWORDS. Innovation, Science and Technology, Patent, Aerospace and Defence.
\end{abstract}

\section{Introduction}

Le 7 juin 2017, la Commission Européenne a présenté sa vision de la «Défense européenne » centrée autour de la recherche scientifique et industrielle. Ce projet est un premier pas pour développer une politique d'innovation de défense commune aux pays membres de l'Union Européenne via une dotation annuelle estimée à 500 millions d'euros par an. Cette avancée dans la coopération européenne en termes de recherche devra s'accompagner de résultats en termes d'innovation. Or, le modèle d'innovation de défense est souvent appréhendé par des modèles linéaires, aujourd'hui remis en cause par l'accélération de la production et de la diffusion de connaissances dans la société permise par la révolution des nouvelles technologies de l'information et de la communication. Un nouveau modèle économique émerge cette révolution : l'économie fondée sur la connaissance (knowledge-based economy) [FOR 98] qui met notamment l'accent sur la place de la recherche scientifique dans le processus d'innovation.

Dans cet article, nous proposons d'évaluer les évolutions du modèle d'innovation du secteur de l'Aérospatial et la Défense (A\&D ci-après) à l'aune de cette vision de l'économie. Nous défendons l'idée que le secteur de l'A\&D ne fait pas figure d'exception face à cette évolution des modèles d'innovation et plus précisément en termes d'utilisation des connaissances scientifiques. Premièrement, nous analysons l'évolution de la contribution des connaissances scientifiques aux innovations 
technologiques de l'A\&D entre 1945 et 2015. Deuxièmement, nous cherchons à connaitre l'influence de cette évolution sur la qualité des innovations de ce secteur.

Afin de réaliser cette étude, nous utilisons l'outil bibliométrique. L'intensité d'utilisation des connaissances scientifiques dans la production de l'innovation est introduite par les travaux de Narin ([NAR 85], [ALB 91] , [NAR 97]). Il étudie l'intensification des relations entre la recherche scientifique publique et la production technologique américaine à travers deux vecteurs : d'une part, via la publication de brevets industriels par des chercheurs académiques, et d'autre part via un comptage de la non patent literature (NPL) présente dans les brevets sur deux périodes (1987-1988 et 1993-1994) [NAR 97]. Leur article montre une augmentation de 30\% des citations NPL dans les brevets entre ces deux périodes, c'est-à-dire une croissance significative de l'utilisation de connaissances scientifiques pour la production d'innovation. De plus, des auteurs, tels [MOW 01] ou encore [SAM 03] mettent en évidence que les brevets citant des connaissances fondamentales sont plus originaux et davantage sensibles d'influencer d'autres catégories technologiques. Selon [TIJ 04], l'analyse de la NPL représente la meilleure approximation des interactions entre la science et la technologie (S\&T) et de la créativité humaine permettant l'innovation industrielle. Nous inscrivons notre analyse des liens entre S\&T dans cette tradition en nous concentrant sur la production de l'A\&D. Nous identifions les brevets déposés dans les domaines technologiques de l'A\&D grâce à la classification de l'Observatoire des Sciences et des Techniques (OST 10) ${ }^{1}$. Ces domaines incluent cinq classes technologiques correspondant aux installations offensives et défensives sur les navires, à l'astronautique, aux explosifs, armes et munitions (respectivement les codes de la classification internationale des brevets B63G, B64G, C06, F41 et F42).

Ainsi, nous proposons, dans une première partie, une revue de la littérature permettant de poser le cadre théorique de notre réflexion et la particularité de notre champ d'application qu'est l'A\&D. Les parties suivantes viseront à tester notre hypothèse théorique pouvant être résumée ainsi : l'émergence de l'économie fondée sur la connaissance se traduit par une augmentation des collaborations entre les producteurs de connaissances scientifiques et industrielles entrainant une production accrue d'innovation technologique, à la fois en quantité et qualité. Nous consacrons ainsi la seconde partie de notre travail à une présentation des données de brevets mobilisées pour cette étude ainsi qu'à une description de la méthode utilisée. Enfin, notre dernière partie traitera de l'évolution des contributions des connaissances scientifiques à l'innovation de l'A\&D ainsi qu'une analyse conjointe de la qualité des brevets et des contributions des connaissances scientifiques à ceux-ci afin d'étudier l'évolution du modèle d'innovation de l'A\&D.

\section{Ancrage théorique}

\subsection{L'innovation de défense}

L'innovation technologique est définie dans le manuel d'Oslo [OEC 05, p. 37] comme « les produits et procédés technologiquement nouveaux ainsi que les améliorations technologiques importants de produits et de procédés qui ont été accomplis. ». Dans la défense, [SEM 15] définit l'innovation comme l'action ou le processus d'introduire de nouvelles idées qui est essentiel pour accomplir les missions de défense. De telles capacités permettent l'exécution des missions de défense de manière effective, sans être compromises par un adversaire potentiel. La nécessité de surpasser les ennemis éventuels signifie que les forces armées doivent constamment améliorer leurs tactiques et matériels. Ainsi, l'industrie de défense a des spécificités propres car elle porte en elle des enjeux stratégiques et de souveraineté [DUP 13].

Aujourd'hui l'innovation de défense implique le développement de solutions caractérisées par un haut degré de complexité, d'intégration et de valeur économique [DIT 06]. Ces solutions intensives en ingénierie, intègrent un grand nombre de technologies et nécessitent un ensemble des compétences complémentaires. Un exemple saillant est l'avion de combat car il nécessite des combinaisons de 
connaissances qui diffèrent drastiquement de l'innovation du secteur civil. En effet, il demande à la fois des compétences en termes de motorisations (moteur à réaction, moteur-fusée), d'électronique (radar, système de navigation, assistant électronique ect.) mais aussi les technologies liées à l'armement (missile air-air, air-sol, bombe guidée laser etc.).

L'innovation utilise deux mécanismes pour atteindre ses objectifs [MAR 91]. Le premier est de créer de la variété en explorant les différents sentiers de création de connaissances (recherche, invention, développement, design, etc.). Le second se concentre sur le choix et l'exploitation des différentes alternatives. Dans le secteur civil, la création de variétés est menée par des entrepreneurs ayant pour objectif le profit consécutif à l'introduction d'un nouveau produit sur le marché. L'innovation suit un processus « classique » où les équipes de R\&D développent le design et le marché fournit une réaction rapide à ce nouveau produit. Dans le domaine de la défense, les motivations et retours suivent un parcours différent. Premièrement, les cycles de vie des équipements sont longs et l'innovation est « générationnelle» [BEL 17]. En effet, le paradigme d'innovation dans la défense est techno-centré : le principe est le développement des capacités par générations successives afin d'éviter toute surprise stratégique (exemple du Mirage 2000 et du Rafale). Pour reprendre l'exemple des avions de combat, le lancement du projet «Rafale» à débuter dans les années 1970, pour aboutir à une première mise en service en 2002 pour la Marine nationale et en 2006 dans l'Armée de l'air. Deuxièmement, le contexte dans lequel le système sera utilisé ne peut être anticipé avec exactitude. A titre d'illustration, les hélicoptères d'attaque Tigre, utilisés par l'armée française, se trouvent inadaptés aux conditions naturelles de certaines zones maliennes. En effet, même si cet hélicoptère est prévu pour une utilisation dans le désert, la finesse du sable au Mali entraine une sur-usure des moteurs due à des problèmes de filtrations ${ }^{1}$.

Les matériels militaires ont trois caractéristiques principales qui influencent fortement l'innovation : i) la concurrence militaire entre Etats ; ii) la production différenciée ; et iii) la complexité technologique [SEM 15]. Tout d'abord, la concurrence sur les matériels militaires se fait sur plusieurs critères : précision, fiabilité, manœuvrabilité, protection, qualité et interopérabilité. De telles exigences demandent un développement complexe de l'offre, nécessitant de mêler la recherche scientifique à la recherche technologique. Ensuite, la production différenciée est définie par le fait que les Etats et leurs forces armées ont des préférences en termes de matériels de guerre, du fait des différences géographiques, des types de conflits potentiels etc. Quand les préférences nationales diffèrent fortement, des achats groupés semblent compromis. Enfin, la complexité des équipements militaires augmentent à chaque nouvelle génération. Elle est due au grand nombre de sous-systèmes, composants et interfaces qui constituent les systèmes d'armement. En effet, alors qu'une automobile contient environ 4000 parties, un système de guidage pour un missile intercontinental en contient plus 19000 [KEL 1995] et un moteur à réaction peut excéder 22000 parties [HOB 98].

Le secteur de la défense entretient donc une relation particulière avec l'innovation technologique. La course à l'armement durant le XXème a entrainé une dynamique d'innovation dans l'ensemble de la société. Un exemple de ces innovations est la localisation GPS, un projet initié en 1960 par l'armée américaine sous l'impulsion du président américain Richard Nixon. Cependant, la fin de la Guerre froide et la baisse du budget militaire associée [FAU 16], ont amené les entreprises productrices de technologies de défense à revoir leur modèle d'innovation et à orienter leur production vers plus de dualité [DEP 14] et ainsi s'adapter aux modèles d'innovation « civils ». Or le modèle d'innovation de défense est souvent appréhendé par des modèles linéaires où l'innovation est perçue comme un processus quasi-externe aux développements des programmes d'armement [GAN 92] et où la contribution des connaissances scientifiques est lointaine et de long terme. Cependant, l'évolution de l'économie et de la société remet en cause cette vision linéaire de l'innovation.

\footnotetext{
${ }^{1}$ Source : Article de Le Point International du 2 juin 2016. http://www.lepoint.fr/monde/mali-les-helicopteres-francais-de-loperation-barkhane-les-pales-du-desert-02-06-2015-1932966_24.php\#
} 


\subsection{L'innovation basée sur la connaissance}

A partir des années 1980, l'utilisation accrue de capital immatériel combinée à la révolution des technologies de l'information et la communication ont donné lieu à l'essor d'une économie fondée sur la connaissance [FOR 98]. Les modèles linéaires d'innovation auparavant utilisés ne semblent plus aptes à capter la réalité des échanges. L'innovation n'émerge plus par grappe, ni par surprise mais est devenue la norme. Elle est nécessaire aux entreprises et devient, dans certains secteurs, le cœur de la concurrence [LEN 02]. Ce changement technologique perpétuel se traduit par une combinaison multilatérale, pluridisciplinaire, et mouvante des recherches scientifiques et technologiques et des méthodes d'invention. En 1996, à Paris, l'OCDE [OEC 96, p.3] déclare que les économies développées «s'appuient de plus en plus sur le savoir et l'information. Le savoir est désormais reconnu comme moteur de la productivité et de la croissance économique, ce qui éclaire sous un nouveau jour le rôle que jouent l'information, les technologies et l'apprentissage dans les performances économiques ». Plus particulièrement, la place de la recherche scientifique dans le processus d'innovation est de plus en plus prédominante. [LEY 95] montre que la mondialisation combinée avec l'émergence de l'économie fondée sur la connaissance remet en question la place de la recherche scientifique dans la société et plus particulièrement sa contribution à l'innovation. La science n'est plus dans sa «tour d'ivoire » avec une contribution à long voir très long terme à l'innovation industrielle. Il met en évidence un modèle où la transmission de connaissances entre la recherche publique et industrielle est dynamique et où les contributions scientifiques peuvent être de court terme.

Cette évolution de l'économie et du processus d'innovation est théorisé par [ETZ 00]. Ils s'inspirent de la théorie évolutionniste de la firme [NEL 85]. Cependant, au lieu de se concentrer sur la co-évolution des firmes et de la technologie, sachant l'architecture des connaissances, ils ajoutent une perspective historique et ainsi endogénéîsent l'architecture de la société. Ce modèle, appelé triple-helix model (THM ci-après), rend compte de l'expansion du rôle des connaissances dans la relation entre l'architecture politique et économique de la société. Historiquement, les interactions systématiques entre le marché et la science remontent à la moitié du XIX ${ }^{\text {ème }}$ siècle et sont appelées la « révolution techno-scientifique » $[\mathrm{BRV}$ 74]. Elle se définit comme la transformation de la science elle-même en capital [NOB 79]. Pour l'historien [BRE 02] la collaboration entre la science et la défense est amorcée en France dès 1793 avec la création de la première unité de recherche publique, chargée de « rechercher et d'éprouver les nouveaux moyens de la Défense ». Ainsi l'A\&D, de par ses caractéristiques de haute technologie, de systèmes complexes et de forte relation entre le décideur public et l'industrie, semble particulièrement pertinente pour aborder ce thème. Le THM en prenant en compte les relations entre la science et le marché, ainsi qu'entre le public et le privé, construit une analyse d'interactions entre la recherche scientifique publique et l'industrie via, notamment l'intervention de l'Etat. Les différentes interactions entre l'industrie, le gouvernement et l'université ont pour effet d'engendrer de nouvelles structures (i.e. centres de recherche public-privé, pôles d'innovation) et de créer de nouvelles mécaniques d'intégration de la connaissance entre les différents acteurs.

Le point de départ de cette analyse est que l'économie évolue vers une utilisation et une intégration des connaissances de plus en plus intensive via une diffusion des connaissances et une collaboration des différents acteurs de la société accrue. Dans cet article, nous cherchons à savoir si ce constat s'applique aussi à l'industrie de l'A\&D malgré ses particularités. L'hypothèse testée dans cet article peut être formulée comme suit :

L'émergence de l'économie fondée sur la connaissance se traduit par une intensification et une codification accrue des collaborations entre les producteurs de connaissances scientifiques et industrielles entrainant une plus grande production d'innovation technologique, à la fois en quantité et qualité.

Pour ce faire, il nous faut étudier les liens et leurs évolutions entre les connaissances scientifiques et technologiques. 


\section{Méthodologie}

A cette fin, nous proposons d'utiliser les données de brevets en combinant deux outils d'analyse : d'une part, la bibliométrie, permettant d'étudier l'évolution des citations de littérature scientifique, et d'autre part, les indicateurs de qualité des brevets, à mettre en corrélation avec l'utilisation intensive de connaissances scientifiques.

\subsection{Approche bibliométrique}

L'émergence de l'économie fondée sur la connaissance amène une nécessité de description des distinctions entre science et technologie (ci-après S\&T), entre université et industrie. Cependant, l'évaluation de l'impact de la recherche sur la production innovante est un des champs d'analyse scientifique les plus difficiles à investiguer empiriquement ([COO 98]; [SAL 01]; [COH 02]). Bien qu'il existe une diversité dans les approches de l'interface entre S\&T, un consensus émerge cependant quant à la méthode d'analyse empirique à utiliser : la bibliométrie. Celle-ci est une étude quantitative des aspects du processus de recherche scientifique comme un système de communication [MIN 15], capable de fournir des mesures du lien entre S\&T aux échelles micro, meso et macro. A un degré micro, elle capture la relation entre une publication scientifique et un brevet, ce qui permet d'identifier la proximité entre différents objets de recherche. A l'échelon sectoriel (ou meso), elle capte les transferts de connaissances et les potentiels effets induits (description des bases de connaissances associées à une technologie ou à une industrie particulière). Enfin, le niveau macro permet de construire des tables de concordance entre les classifications scientifiques et les nomenclatures technologiques, d'investiguer les dépendances et de développer des instruments de politique publique.

La bibliométrie est un outil aux multiples facettes ayant pour objectif d'identifier, de quantifier et d'analyser la production des connaissances et leurs qualités. Majoritairement utilisée pour l'étude de la littérature scientifique, cette méthode permet aussi l'analyse des voies de transmission des connaissances entre la recherche scientifique et technologique. Les flux de connaissances permettant l'innovation sont divers: science vers science, technologie vers technologie, technologie vers science et science vers technologie. Narin, à partir des années 1960, consacre ses recherches à l'analyse de la production et la diffusion des connaissances, en introduisant une analyse de co-citation, à l'intérieur de la recherche scientifique (via les publications scientifiques), de la R\&D technologique (via les brevets) mais aussi les flux entre ces deux supports. La production de connaissances de la science peut être approchée par les connaissances contenues dans la littérature scientifique et celle de la technologie par les connaissances diffusées dans les brevets.

La méthode d'analyse des citations entre différents supports de la connaissance est la plus utilisée et c'est celle-ci que nous retenons dans notre étude. Présentée par [NAR 82], elle consiste à étudier les références à des articles scientifiques (non-patent literature, ci-après NPL) dans les brevets et inversement. Selon [TIJ 04], l'analyse de la NPL représente la meilleure approximation des interactions entre S\&T et de la créativité humaine permettant l'innovation industrielle. "Si la littérature scientifique reflète les activités scientifiques, un catalogue pluridisciplinaire des citations peut fournir une vision intéressante de ces activités. Cette vision peut mettre en lumière à la fois « la structure de la science et le développement des processus scientifiques » [GAR 79 p.62]. Cette analyse peut être étendue aux brevets. Dès le début des années 1980, [CAR 81a] montrent l'intérêt de l'analyse de la NPL pour étudier les processus d'innovation. Ils mettent en lumière la dépendance de l'évolution technologique à la recherche scientifique. D'autres auteurs, comme [LET 07] s'intéressent à des domaines technologiques précis. Ils montrent une relation positive entre l'intensité scientifique des brevets et la productivité technologique dans le secteur de la biotechnologie. Cette approche permet aussi des analyses en termes de flux de connaissances sur une zone géographique donnée, [VER 03] regardent la distribution géographique des NPL dans le secteur de la biotechnologie et des technologies de l'information et mettent en évidence une concentration à l'échelle nationale des citations ainsi qu'une contribution significative des connaissances produites aux Etats-Unis et en Europe pour l'innovation 
technologique dans le monde. Dans une autre optique, [LOO 03] expliquent la performance à l'innovation par l'intensité d'utilisation de références scientifiques dans les brevets. Afin d'affiner ce type d'analyse, nous pouvons identifier trois catégories de citations : i) autocitation [NAR 97], ii) citations nationales et les citations internationales [HIC 01]. [NAR 97] met ainsi en évidence les biais de cette analyse en pointant le problème des autocitations (self-citations), des barrières linguistiques amenant les chercheurs à préférer des références nationales (qui ne sont pas forcément les plus pertinentes), et des problèmes de connaissances des déposants de brevets et des auteurs d'articles scientifiques sur la production des types de recherche. Contrairement à d'autres indicateurs de coopération entre la recherche scientifique et industrielle telles que des collaborations informelles ou encore de chercheurs issus du milieu académique travaillant pour l'industrie, l'étude de la NPL montre des connexions explicites, volontaires, sélectives et asymétriques. Cette méthode permet d'étudier le secteur de l'A\&D qui tient une place particulière dans les systèmes d'innovation.

\subsection{Source et analyse des données}

\subsubsection{La qualité des brevets}

Cette étude de la NPL dans les brevets de l'A\&D doit être compléter par l'analyse de l'influence de la diffusion des connaissances scientifiques sur la qualité de l'innovation. En effet, la vision de l'économie fondée sur la connaissance défend l'hypothèse que la collaboration accrue entre les différents acteurs producteurs de connaissances accélère et améliore le processus d'innovation. Trois arguments principaux permettent d'expliquer comment la science contribue à l'innovation de l'industrie :

-En lui permettant d'éviter des expériences non pertinentes et de se concentrer sur les voies de recherche pertinentes [FLE 04].

-En augmentant et en améliorant la capacité d'absorption interne et externe des nouvelles connaissances par la firme [COC 98].

- En améliorant la qualité et la valeur économique des technologies produites par les entreprises [ARO 94].

Dans cet article, nous nous intéressons à la contribution de la science à la qualité de l'innovation du secteur de l'A\&D. La qualité d'un brevet peut être appréciée à l'aide de plusieurs critères. Le premier critère est celui de la valeur « commerciale ». Dans ce cas, la qualité d'un brevet est traitée comme la valeur économique du brevet sur le marché, i.e. l'augmentation de valeur d'un portefeuille de brevets d'une firme consécutive au dépôt du brevet. D'autre part, la qualité d'un brevet peut-être vue sous l'angle « technologique ». Dans ce cas, la qualité du brevet dépend d'un arbitrage entre la généralité et originalité. Afin d'étudier cette question de la qualité, à la fois sous l'angle commercial et technologique, nous mobilisons quatre indicateurs de qualité des brevets, discutés par le rapport de l'OCDE [SQU 13].

Premièrement, la qualité commerciale d'un brevet peut être approchée par la taille de la famille de brevets (patent family size), notée NPN. Rappelons qu'un groupe de brevets protégeant la même innovation est appelé une famille de brevets. Ici, la valeur du brevet est associée à la portée géographique de la protection de l'innovation [LAN 98]. [HAR 03] montrent qu'une famille de brevets ayant des représentations dans beaucoup de pays est un indicateur de la valeur économique du brevet. En effet, étendre la protection d'une innovation à plusieurs pays dans le monde traduit, de la part des déposants, une disposition à accepter des coûts de brevetage ainsi que des délais administratifs supplémentaires. Or ces coûts supplémentaires ne sont supportés que si la firme, ou le déposant, estime qu'il en retirera un bénéfice supérieur.

Deuxièmement, la généralité d'un brevet peut être appréciée par le patent scope correspondant au nombre de domaines technologiques différents référencés dans la famille de brevets, noté $N B I P C$. En effet, l'examinateur du brevet assigne à chaque brevet un ou plus domaine technologique à 4-digit basé 
sur la classification internationale des brevets (WIPO). Cet indicateur permet de capter la généralité de l'innovation et ainsi que son influence sur le marché. [LER 94] observe que la technologie contenue dans les portefeuilles de brevets des firmes affecte significativement la valeur de la firme sur le marché. L'idée supportant cet argument est que les brevets sont plus précieux lorsque de nombreux substituts dans la même classe de produit sont disponibles et que ceux-ci sont possédés par la même firme. Cet argument est d'autant plus vrai lorsque les innovations influencent potentiellement différents secteurs industriels.

Troisièmement, l'originalité d'un brevet peut être approximée par le nombre de citations de brevets (backward citation) dans le brevet, notée NSCT. En vue d'évaluer la nouveauté d'une innovation, un inventeur doit citer tous les brevets précédents reliés à son invention. L'examinateur du brevet doit s'assurer que tous les brevets appropriés sont cités. Ceux-ci permettent d'attester de la brevetabilité de l'invention et définir la légitimité de la revendication de paternité. [CRI 08] expliquent qu'un indicateur basé sur le nombre de citations dans un brevet atteste du degré de nouveauté de l'invention et évalue les transferts de connaissances réalisés pour produire cette invention. De plus, agréger les citations de brevets à l'échelle d'une technologie permet d'éclairer le processus d'innovation et ses dynamiques. [HAR 03] trouvent une corrélation positive entre les nombre de brevets cités et la valeur d'un brevet sur le marché. D'autre part, cet indicateur permet de mettre en lumière la nature du processus d'innovation, i.e. si l'invention est incrémentale ou de rupture. Ainsi, lorsque le nombre de brevets cités est faible, il est aisé de conclure que l'invention technologique concernée ne se repose pas sur des innovations passées, et sera donc plutôt de rupture. A l'inverse, lorsque la quantité de brevets cités est importante, cela s'interprète comme une invention reposant sur nombre d'innovations passées, et sera donc de nature incrémentale. Afin d'utiliser un tel indicateur, il est primordial de contrôler les autocitations des inventeurs. En effet, les autocitations constituent à la fois un biais dû à un comportement stratégique de la part de l'inventeur - l'inventeur peut s'auto-citer afin d'augmenter les statistiques de qualité sur ces brevets - ainsi qu'un biais de connaissance - l'inventeur se cite car c'est ce qu'il connait. C'est pourquoi, nous ne considérons ici les citations, à l'exclusion des autocitations.

Enfin, les brevets citant, noté NCTG (forward citation) traduisent à la fois l'importance technologique du brevet et la valeur économique et commerciale de l'invention ([TRA 90]; [HAR 03]; [HAL 05]). Le nombre de brevets citant traduit l'importance technologique. L'idée est que l'innovation contenue dans le brevet entraine une grappe d'innovations incrémentales. La quantité de brevets citant marque aussi la valeur économique d'un brevet en indiquant à l'ensemble du secteur industriel la potentialité de l'innovation et ainsi augmente la valeur de marché de la firme déposante.

A partir de cet ensemble d'indicateurs de qualité des brevets, nous cherchons à connaître le lien qui existe entre ceux-ci et les changements de dynamique d'innovation, traduit par la quantité de NPL contenue dans les brevets (notée NPL/brevet).

\subsubsection{Les brevets}

Afin de construire notre évaluation de la contribution des connaissances scientifiques à l'innovation technologique, nous utilisons les brevets comme proxy de l'innovation technologique du secteur de l'A\&D. Comme cité précédemment, les brevets sont des bonnes estimations de l'innovation technologique [GAR 79]. En effet, toute organisation qui parvient à se saisir d'opportunités technologiques se trouve confrontée à la question de la protection de son avance [TEE 88]. Pour cela, il existe différents régimes de protection possibles parmi lesquels le brevet, particulièrement performant pour s'approprier une innovation de produit et le secret qui, quant à lui, ferait plus facilement valoir ses droits sur des innovations de procédés. L'arbitrage entre ces deux modes de protection de l'innovation se fait par l'organisation qui en est à l'origine. Cependant, dans le cas d'innovations ayant trait à la défense, l'Etat peut contraindre une organisation à tenir le secret, alors que celle-ci aurait pourtant préféré publier un brevet. En effet, au regard des enjeux de souveraineté et alors même que le potentiel 
de diffusion d'une technologie peut être considéré comme élevé, le gouvernement est en capacité d'intervenir pour limiter le périmètre d'exploitation économique d'une innovation. $\mathrm{Ce}$ biais d'information créé par l'existence du secret comme protection de la propriété intellectuelle constitue une limite malheureusement infranchissable à l'analyse empirique de l'innovation. En effet, la nature même de ce biais, le secret, explique qu'il ne peut être observé. C'est pourquoi nous utilisons comme approximation de l'innovation les brevets, qui comme tout indicateur est imparfait mais qui est aussi le plus complet auquel les chercheurs peuvent avoir accès. Il est un document très informatif avec des données à la fois sur les détenteurs, sur la nature de l'innovation (domaine technologique) et les connaissances mobilisés pour la produire. De plus, ce document juridique harmonisé, contrôlé et référencé par des experts des domaines technologiques, est plus fiable et détaillé que n'importe quelle donnée sur l'innovation.

Les brevets sont des droits de propriété industrielle assurant une protection dans un ou plusieurs pays donnés. Il est fréquent que les inventions fassent l'objet de plusieurs demandes de brevets auprès de différents offices nationaux ou régionaux des brevets ou auprès de l'Organisation mondiale de la propriété intellectuelle (WIPO). Le WIPO est l'instance mondiale pour les services, les politiques, l'information et la coopération en matière de propriété intellectuelle. C'est pourquoi l'analyse en termes de famille de brevets est plus pertinente lorsque l'on s'intéresse à l'innovation. En effet, la famille de brevets permet de capter une innovation effective et non simplement la reproduction d'une innovation dans un contexte national ou régional. Orbit-Questel ${ }^{2}$ a développé une définition de la famille qui combine la règle de famille stricte de l'European Patent Office (EPO) ${ }^{3}$ avec des règles complémentaires qui permettent de prendre en compte les liens avec la demande parente EPO et/ou PCT, les liens entre les demandes américaines provisoires et les demandes américaines publiées. Cette définition prend également en considération les définitions de la notion d'invention différentes selon les offices.

Pour étudier les technologies contenues dans les brevets, nous utilisons la classification internationale des brevets du WIPO, qui est unanimement reconnue et utilisée pour l'étude des brevets. Elle divise la technologie en huit sections comptants environs 70000 subdivisions au niveau le plus désagrégé. Nous utilisons la base de données brevets générée par Orbit. Entre 1945 et 2015, nous avons identifié 51161 familles de brevets contenant les domaines technologiques de l'A\&D, déposées dans plus de 63 offices de dépôts dans le monde (voir annexe 2 pour la distribution des familles de brevets par premier office de dépôt).

\section{Résultats}

Dans cette partie, nous présentons les résultats empiriques de notre étude. Premièrement, est développé une analyse descriptive des données de famille de brevets du domaine $\mathrm{A} \& \mathrm{D}$, en particulier un compte rendu de l'utilisation de connaissances scientifiques dans ces familles. Dans un second temps, nous approfondissons cette étude descriptive en introduisant les corrélations entre la qualité des brevets et la NPL.

\subsection{Analyse descriptive}

L'étude de ces familles de brevets et de leurs données associées permet de mettre en évidence des grandes tendances. Dans un premier temps, nous observons une forte augmentation dans le temps des dépôts de familles de brevets, ce qui est conforme à la littérature (e.g. [KOR 99] [KOR 00]; [HAL 01] ; [HAL 04]; [JIN 04]. Cette tendance s'explique par une combinaison de facteurs. Premièrement, l'entrée de nouveaux pays sur le «marché des brevets » notamment la Chine et la Corée du Sud (WIPO, 2016). Deuxièmement, [KOR 99] l'expliquent par la combinaison de l'augmentation globale de l'innovation dans la société et l'amélioration du management de la R\&D. Troisièmement, l'augmentation de l'importance des portefeuilles de brevets en vue de se protéger de ses concurrents a contribué à cette tendance [HAL 03]. En effet, la valeur accrue que les marchés financiers accordent aux portefeuilles de 
brevets amène les entreprises, notamment les nouvelles firmes à verrouiller leurs innovations technologiques grâce au brevetage.

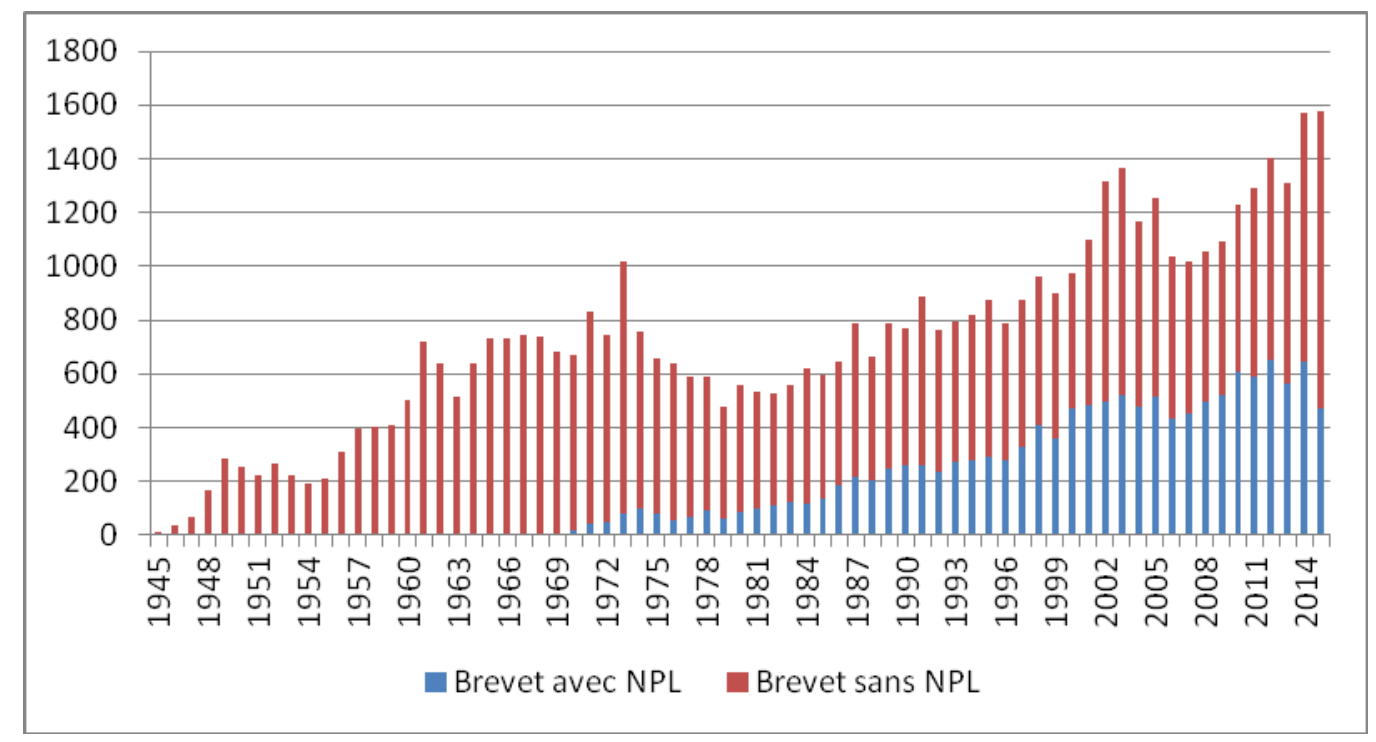

Figure 1. L'évolution des brevets et de la NPL de l'A\&D entre 1945 et 2015 Source : Auteur à partir des données Orbit

D'autre part, le référencement de contributions scientifiques à l'innovation contenue dans le brevet, connait elle aussi une forte hausse. Comme l'illustre la figure 1, la première référence à la littérature scientifique dans nos brevets intervient au début des années 1970. Ces références contiennent à la fois des articles publiés dans des revues à comité de lecture mais aussi des livres scientifiques ainsi que des compte rendus de conférences. Cette apparition de la NPL dans le brevet ainsi que sa hausse constante peut s'expliquer par trois éléments principaux. Premièrement, les offices de dépôts augmentent leurs exigences en termes de complétude des brevets. Deuxièmement, le travail des experts des offices de dépôts chargés de référencer les brevets est de plus en plus précis. Enfin, notre hypothèse de départ qui suppose que le changement du modèle d'innovation a mené à une croissance des innovations technologiques poussée par une diffusion accrue des connaissances scientifiques. Comme nous l'avons présenté précédemment la rencontre entre la montée du capital immatériel et la révolution des technologies de l'information et la communication a donné lieu à l'essor d'une économie fondée sur la connaissance [FOR 98]. Le changement technologique perpétuel qui en résulte se traduit par une combinaison multilatérale, pluridisciplinaire, et mouvante des recherches scientifiques et technologiques et des méthodes d'invention. Ces nouvelles tendances sont associées à une augmentation de l'utilisation des connaissances générées par les recherches universitaires [BRN 05]. Selon l'OCDE [OEC 96], la part de la haute technologique dans la production, largement dépendante de la production et de la diffusion des connaissances notamment scientifiques, a doublé entre 1970 et 1990 dans la production industrielle et les exportations, et cette évolution est se perpétue aujourd'hui. Ce constat de l'OCDE est conforté par notre étude des secteurs technologiques de l'A\&D. La figure 1 montre en effet une part croissante des brevets faisant référence à au moins une littérature scientifique. Le nombre de brevets citant de la NPL passe de quasi-existant avant les 1980, à plus du tiers des brevets à partir des années 2000 . 


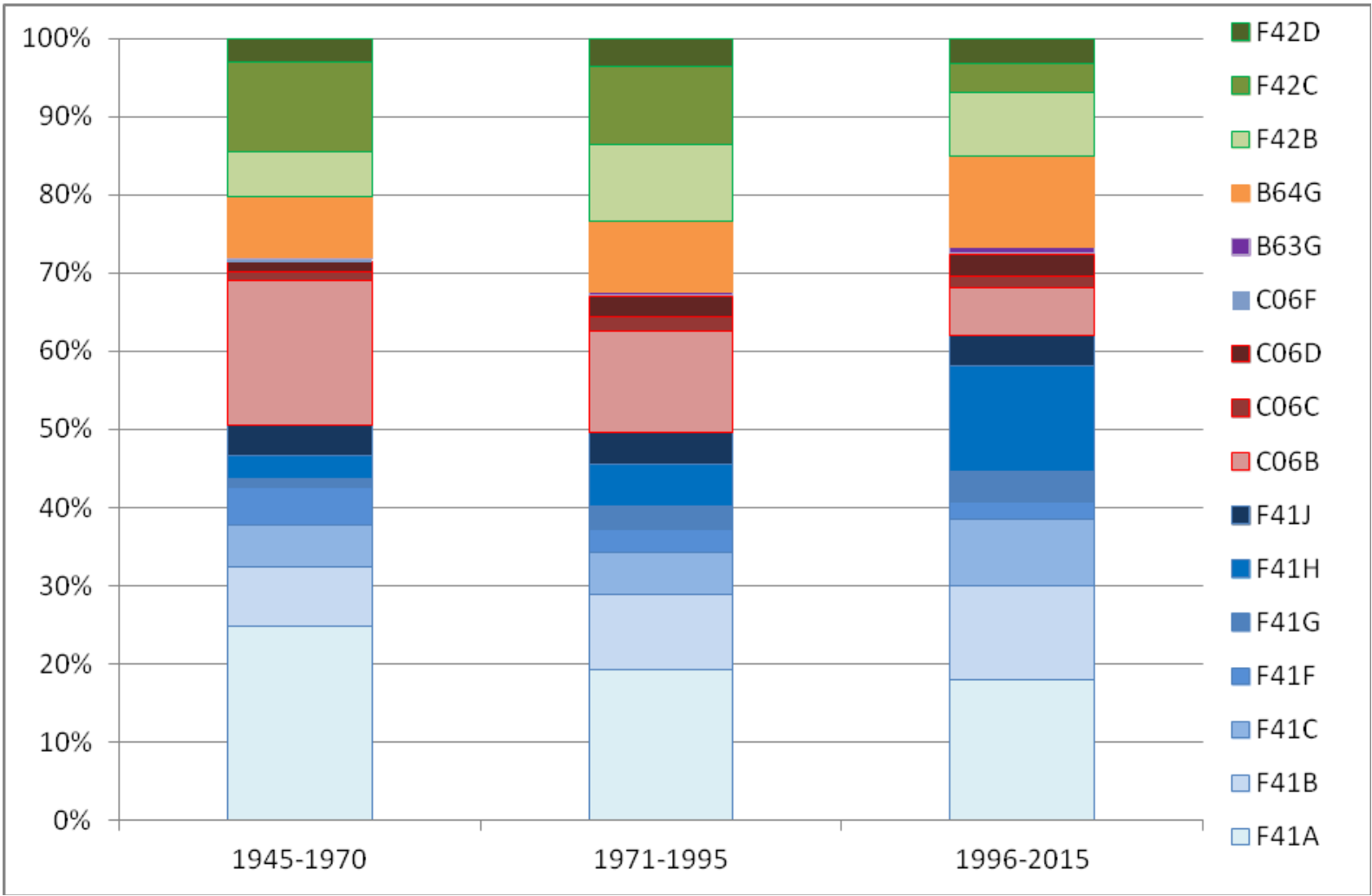

Figure 2. Répartition des brevets A\&D par domaines technologiques entre 1945 et 2015 Source : Auteur à partir des données d'Orbit

Les brevets de l'A\&D sont composés de cinq classes technologiques distincts et leur poids dans le domaine évolue au cours de la période. Ainsi, la figure 2 montre que la technologie des armes (F41) domine le domaine A\&D sur l'ensemble de la période avec un poids allant jusqu'à $60 \%$ sur la dernière période (1996-2015). L'augmentation ce poids est tirée par le blindage et autres moyens d'attaque ou de défense, tel que le camouflage. Si l'économie fondée sur la connaissance est une analyse adéquate de notre société alors ce domaine technologique, du fait de son expansion, devrait être intensif en utilisation de connaissances scientifiques. C'est ce que nous proposons d'étudier dans la figure 3.

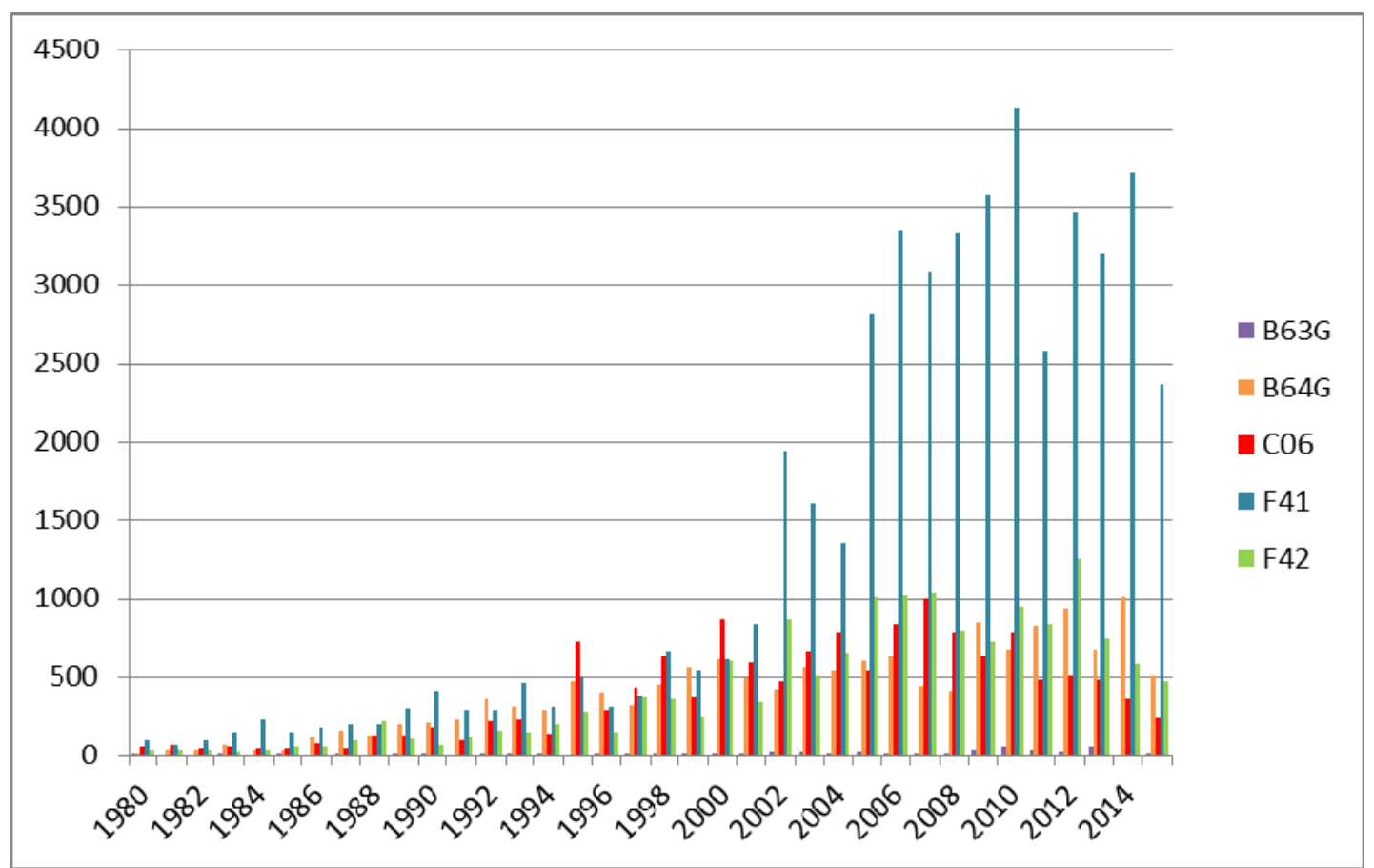

Figure 3. Evolution de la NPL en volume par domaine technologique d'A\&D Source : Auteur à partir des données d'Orbit 
Afin de rendre les résultats plus lisibles, nous proposons d'analyser les classes technologiques regroupées au niveau 3-digit pour F41, F42 et C06. Nous présentons cette analyse à partir de $1980 \mathrm{car}$ c'est à partir de cette période que la présence de la NPL dans les brevets émerge dans l'ensemble des domaines technologiques étudiés. La figure 3 montre que l'augmentation de la part de F41 dans l'ensemble des familles de brevets du domaine A\&D s'accompagne d'une hausse plus que significative des citations de NPL de cette classe. En effet, même si la quantité de NPL augmente dans l'ensemble des classes technologiques sur la période 1980-2015, ce sont les technologies se rapportant à F41 qui observent la plus forte augmentation en volume de NPL, notamment à partir de 2002. Cependant, cette étude en termes de volume ne permet pas de prendre en compte l'augmentation de la NPL relativement au poids de la classe technologique dans le domaine de l'A\&D.

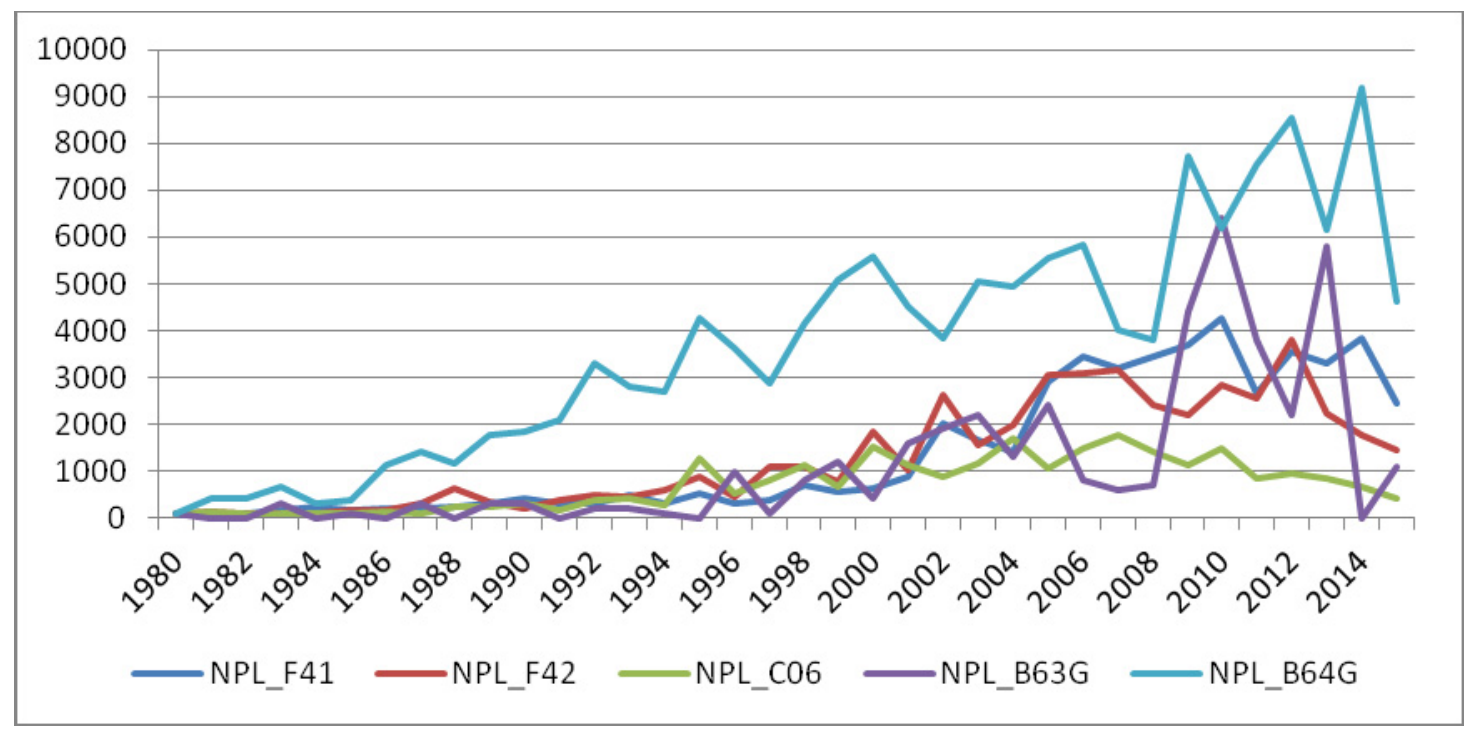

Figure 4. Evolution de la NPL par domaine technologique d'A\&D (base 100 : 1980) Source : Auteur à partir des données Orbit

C'est pourquoi nous présentons la figure 4 qui illustre l'évolution du nombre de référence NPL dans les brevets par technologie de l'A\&D, en base 100 de l'année 1980. Nous observons que l'ensemble des technologies a connu une forte de croissance de la NPL sur la période 1980-2015. Cette croissance est la plus marquée pour le domaine technologique B64G, correspondant à l'innovation technologique des véhicules et équipements pour l'astronautique. Cette catégorie comprend notamment l'ensemble des véhicules spatiaux, les outils adaptés au travail dans l'espace, les vêtements spatiaux ou encore les simulateurs de conditions de vie dans l'espace. A titre d'illustration, un des brevets classés dans cette catégorie est un lanceur de satellites, publié en 1990 et citant comme NPL un article scientifique publié dans le Journal of the British Interplanetary Society en 1977. Les autres domaines technologiques étudiés ont eux aussi connus une croissance marquée de la quantité de NPL dans leurs brevets, avec une tendance globale à l'accélération de cette augmentation. En effet, entre 1980 et 2010, la quantité de NPL dans les brevets associés aux installations offensives et défensives des navires (B63G) a été multipliée par 60. Nous observons la même tendance pour les technologies des armes et des munitions (respectivement F41 et F42). Pour l'innovation se rapportant à la chimie, et plus particulièrement aux explosifs (C06), contrairement aux autres domaines, la croissance de la NPL dans les brevets est moins erratique et a été multipliée par 8 entre 1980 et 2015.

Ainsi, cette étude descriptive des données de brevets et de leurs citations en termes de NPL met en évidence l'augmentation conjointe de la mobilisation de connaissances scientifiques dans l'industrie et de la production d'innovation technologique. Cependant, cette étude descriptive n'est pas suffisante pour comprendre comment l'intensification de l'utilisation de connaissances scientifiques dans la production d'innovation technologique influence la capacité d'innover, notamment en termes qualitatif. A cette fin, nous proposons dans la partie suivante d'étudier le lien entre la quantité de NPL dans les 
familles de brevets et la qualité de ces mêmes brevets par classe technologique appartenant au domaine de l'A\&D.

\subsection{Connaissances scientifiques et qualité de l'innovation technologique}

La figure 5 représente les tables de corrélations entre la quantité de NPL et les indicateurs de qualité des brevets. Les coefficients de corrélation (de Pearson) ont été calculés à partir de 1980 et jusqu'en 2015. Ces tables de corrélation montrent plusieurs éléments. Globalement, nous observons une corrélation positive et significative entre la quantité de NPL dans les brevets et les différents indicateurs de qualité, malgré une faiblesse des coefficients (en moyenne entre 0,2 et 0,3 ) pour la majorité des observations. Cependant, une corrélation sort particulièrement du lot pour l'ensemble des classes technologiques : la corrélation entre la quantité de NPL et de brevets cités $(0,6)$. Cette tendance peut s'expliquer par deux facteurs. D'une part, l'amélioration du renseignement contenu dans les brevets est due à la fois à la vigilance accrue des examinateurs des brevets et au rôle croissant des services de propriété intellectuelle dans les firmes innovantes. D'autre part, elle met en évidence l'émergence des collaborations accrues entre les producteurs de connaissances scientifiques et technologiques. En effet, nous observons qu'une hausse des brevets cités, traduisant la nature incrémentale de l'innovation, augmente la quantité de NPL présent dans ce même brevet. Comme nous l'avons développé dans la partie méthode, une quantité importante de brevets cités traduit la nature incrémentale de l'innovation. Ainsi, cette corrélation entre NPL/brevet et NSCT peut s'interpréter comme le fait que le caractère incrémental des innovations de l'A\&D est non seulement basé sur les innovations technologiques antérieures mais aussi sur les connaissances scientifiques produites. On observe que ce lien est particulièrement fort pour le domaine technologique regroupant les armes (F41).

Ensemble des brevets A\&D

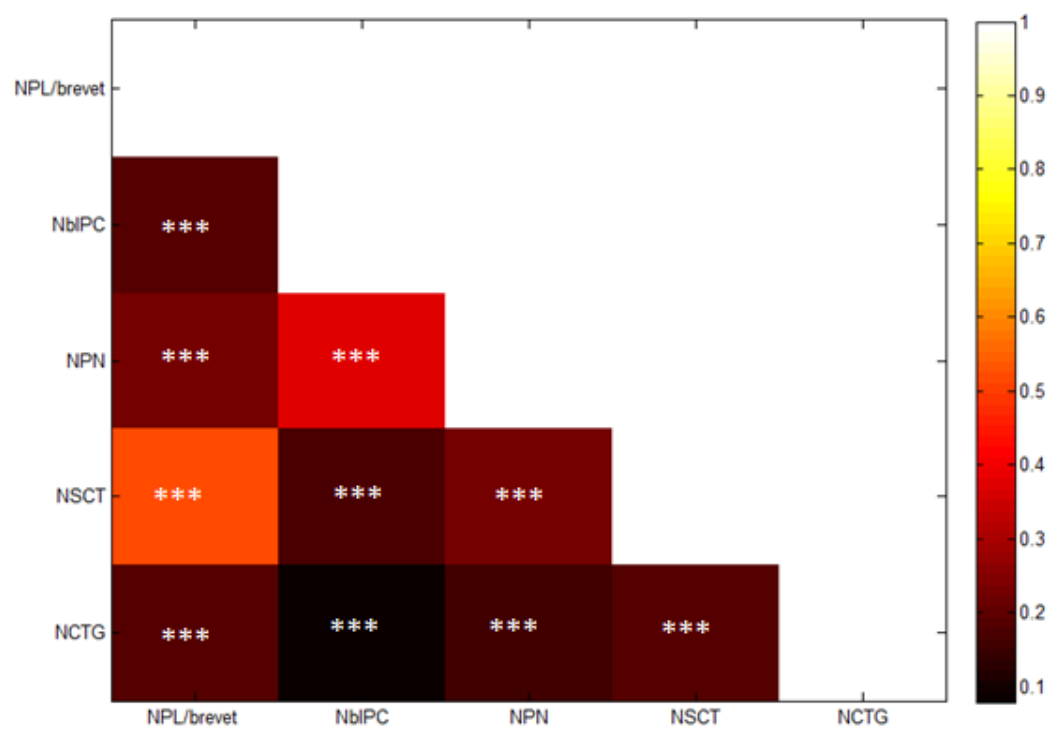



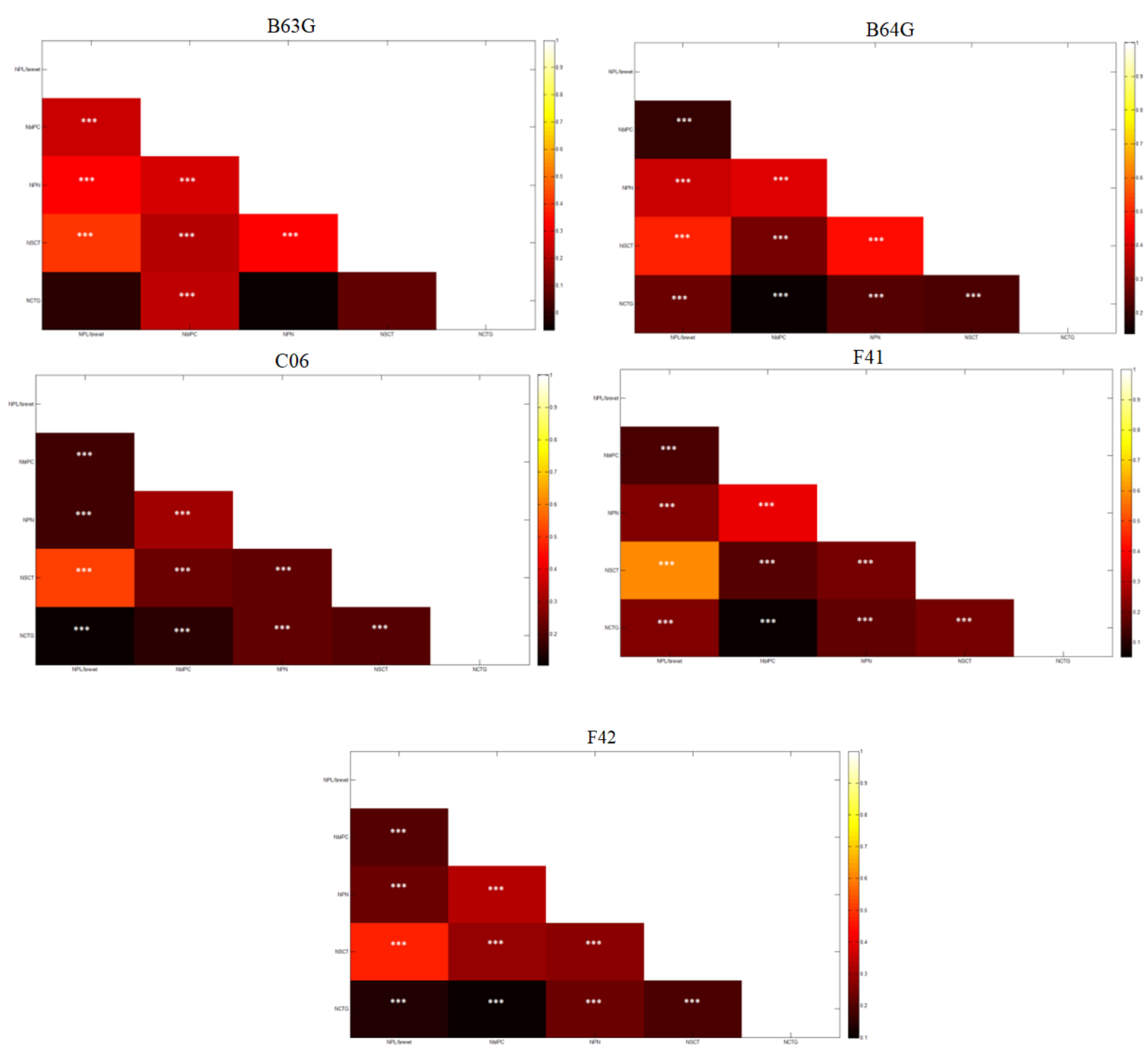

Figure 5. Tables de corrélation entre contribution scientifique et qualité des brevets entre 1980 et 2015 par domaines technologiques

Si l'on s'intéresse aux résultats par domaines technologiques, nous remarquons que seule la technologie B63G, i.e. les installations offensives ou défensives sur les navires, sous-marins et porteavions, a des coefficients différents de ceux du tableau global. En effet, le coefficient de corrélation entre la NPL/brevet et le NbIPC, la taille de la famille et le nombre de brevets cités est plus haut que pour les autres domaines technologiques (entre 0,3 et 0,4 ) alors que celui entre la NPL/brevet et le nombre de brevet citant n'est pas significatif. Cette technologie est très spécifique car caractérisée par une forte composante militaire et ainsi qu'un domaine d'action plutôt restreint : le naval.

Ainsi, cette étude des coefficients de corrélation a permis de mettre en évidence un lien linéaire assez faible, bien que significatif, entre la qualité des brevets et la quantité de NPL présente dans ceuxci. Ceci vient confirmer notre hypothèse théorique visant à défendre que l'émergence de l'économie fondée sur la connaissance se traduit par une augmentation des collaborations entre les producteurs de connaissances scientifiques et industrielles, entrainant une production accrue d'innovation technologique en termes de qualité. De plus, cette tendance à l'augmentation de la NPL met en évidence un changement dans la nature de l'innovation de l'A\&D incrémentale, qui repose de plus en plus sur les connaissances scientifiques. 


\section{Conclusion}

Dans cet article, nous avons évalué les modifications du modèle d'innovation du secteur de l'A\&D sous l'angle de la contribution des connaissances scientifiques. Dans un premier temps, nous avons analysé l'évolution de la contribution des connaissances scientifiques aux innovations technologiques de l'A\&D entre 1945 et 2015. Cela a permis de mettre en évidence une augmentation de la NPL présente dans les brevets, avec une émergence de ce type de citation à partir des années 1980. Cette apparition correspond au début de ce que les économistes appellent l'« économie fondée sur la connaissance $»$. Ainsi, nous pouvons conclure que le secteur de l'A\&D ne fait pas exception quant aux changements de modèles d'innovation observés dans l'ensemble de l'économie. Cela vient ainsi conforter l'argument de [COW 95] qui remettent en cause la spécificité linéaire du modèle d'innovation militaire défendu par [GAN 92]. Deuxièmement, nous avons approfondi ce sujet à travers l'étude de la qualité des brevets et de son lien avec la NPL. Cette analyse a mis en évidence la co-évolution des citations de brevets et de NPL dans l'ensemble du secteur A\&D, ainsi que la particularité du domaine technologique B63G, englobant les équipements offensifs et défensifs des navires, les sous-marins et les porte-avions. Cependant, notre analyse est biaisée par la sélection, a priori, des domaines technologiques ayant trait à la défense sans traiter de la question de la dualité de telles innovations. Cette étude quantitative de l'innovation a ainsi mis en lumière l'émergence de l'économie fondée sur la connaissance se traduisant par une augmentation des collaborations entre les producteurs de connaissances scientifiques et industrielles. Il conviendrait, à l'avenir, de réaliser une étude similaire en sélectionnant, non pas les domaines technologiques A\&D mais les firmes ayant une activité de défense.

\section{Bibliographie}

[ALB 91] ALBERT M.B., AVERY F., NARIN F., McALLISTER P., 1991. «Direct Validation of Citation Counts as Indicators of Industrially Important Patents ». Research Policy 20 (3): 251-59.

[ARO 94] ARORA A., GAMBARDELLA A., 1994. « The changing technology of technological change: general and abstract knowledge and the division of innovative labour». Research Policy, Special Issue in Honor of Nathan Rosenberg, 23 (5): 523-32.

[BEL 17] BELLAIS R., DROFF J., « Innovation, Technology and Defense Procurement: Reform or Paradigmatic Shift? » dans K. BURGESS et P. ANTILL, Emerging Strategies in Defense Acquisitions and Military Procurement, IGI Global, Hershy, 2017, 205-22.

[BRN 05] BRANSTETTER L., OGURA Y., 2005. «Is Academic Science Driving a Surge in Industrial Innovation? Evidence from Patent Citations ». Working Paper 11561. National Bureau of Economic Research. http://www.nber.org/papers/w11561.

[BRV 74] BRAVERMAN H., 1974. Labor and Monopoly Capital: the degradation of work in the twentieth century. Monthly Reviw Press New York.

[BRE 02]BRET P., 2002. «L'État, l'armée, la science. L'invention de la recherche publique en France (1763-1830)». Annales historiques de la Révolution française 328 (1): 278-278.

[CAR 81a] CARPENTER, M.P., NARIN F., WOOLF P., 1981a. « Citation Rates to Technologically Important Patents ». World Patent Information 3 (4): 160-63.

[CAR 81b] - 1981b. « Citation rates to technologically important patents ». World Patent Information 3 (4): 16063.

[COC 98] COCKBURN I.M., HENDERSON R.M., 1998. "Absorptive Capacity, Coauthoring Behavior, and the Organization of Research in Drug Discovery ». The Journal of Industrial Economics 46 (2): 157-82.

[COH 02] COHEN, W.M., NELSON R.R., WALSH J.P., 2002. "Links and Impacts: The Influence of Public Research on Industrial R\&D.” Management Science 48 (1): 1-23.

[COO 98] COOMBS R., HULL R., 1998. « Knowledge Management Practices and Path-Dependency in Innovation » 27.

[COW 95] COWAN, R., FORAY D., 1995. « The Changing Economics of Technological Learning ». Monograph. mai 1995. http://pure.iiasa.ac.at/4553/. 
[CRI 08] CRISCUOLO P., VERSPAGEN B,. 2008. «Does it matter where patent citations come from? Inventor vs. examiner citations in European patents ». Research Policy, Special Section Knowledge Dynamics out of Balance: Knowledge Biased, Skewed and Unmatched, 37 (10): 1892-1908.

[DEP 14] DEPEYRE C., 2014. « Boeing Boeing: la dualité civil-militaire source d'un rebond stratégique dans l'ère postguerre froide, "Boeing Boeing": the civil-military balance, source of a strategic bounce in the post cold war era ». Entreprises et histoire, no 73 (mai): 58-74.

[DIT 06] DITTRICH K., FERDINAND J., van der VALK W., WYNSTRA F., 2006. "Dealing with dualities ». Industrial Marketing Management, IMP 2005: Dealing with Dualities, 35 (7): 792-96.

[DUP 13] DUPUY R., 2013. "L'industrie européenne de défense: changements institutionnels et stratégies de coopétition des firmes ». Innovations 42 (3): 85. https://doi.org/10.3917/inno.042.0085.

[ETZ 00] ETZKOWITZ H., LEYDESDORFF L., 2000. "The dynamics of innovation: from National Systems and "Mode 2" to a Triple Helix of university-industry-government relations ». Research Policy 29 (2): 109-23.

[FAU 16] FAUCONNET C., MALIZARD J., 2016. «Rétrospective des exportations d'armement en France (19582015) ». Revue Défense National, 2016.

[FLE 04] FLEMING L., SORENSON O., 2004. « Science as a Map in Technological Search ». Strategic Management Journal 25 (8-9): 909-28.

[FOR 98] FORAY D., LUNDVALL B., 1998. « The Knowledge-Based Economy: From Economics of Knowledge to the Learning Economy ». In The Economic Impact of Knowledge. Routledge.

[GAN 92] GANSLER, J.S., 1992. « Restructuring the Defense Industrial Base ». Issues in Science and Technology 8 (3): $50-58$.

[HAL 04] HALL, B.H., 2004. « Exploring the Patent Explosion ». The Journal of Technology Transfer 30 (1-2): 35-48.

[HAL 05] HALL, B.H., JAFFE A., TRAJTENBERG M., 2005. " Market Value and Patent Citations ». The RAND Journal of Economics 36 (1): 16-38.

[HAL 01] HALL, B.H., ZIEDONIS R.H., 2001. « The Patent Paradox Revisited: An Empirical Study of Patenting in the U.S. Semiconductor Industry, 1979-1995 ». The RAND Journal of Economics 32 (1): 101-28.

[HAR 03] HARHOFF D., SCHERER F.M., VOPEL K., 2003. "Citations, family size, opposition and the value of patent rights ». Research Policy 32 (8): 1343-63.

[HIC 01] HICKS D., BREITZMAN T., OLIVASTRO D., HAMILTON K., 2001. « The changing composition of innovative activity in the US - a portrait based on patent analysis ». Research Policy 30 (4): 681-703.

[HOB 98] HOBDAY M., 1998. «Product complexity, innovation and industrial organisation ». Research Policy 26 (6): 689-710.

[KEL 95] KELLEY M.R., WATKINS T.A., 1995. « In from the Cold: Prospects for Conversion of the Defense Industrial Base ». Science 268 (5210): 525-32.

[JIN 04] JINYOUNG K., MARSCHKE G., 2004. " Accounting for the recent surge in U.S. patenting: changes in R\&D expenditures, patent yields, and the high tech sector ». Economics of Innovation and New Technology 13 (6): 543-58.

[KOR 99] KORTUM S., LERNER J., 1999. "What is behind the recent surge in patenting?1The paper is a condensed version of 'Stronger Protection or Technological Revolution: What is Behind the Recent Surge in Patenting?' Carnegie-Rochester Conference Series on Public Policy, Vol. 48, 1998, 247-304.1 ». Research Policy 28 (1): 1-22.

[KOR 00]_. 2000. Assessing the Contribution of Venture Capital to Innovation. Vol. 31.

[LAN 98] LANJOUW J.O., PAKES A., PUTMAN J., 1998. « How to Count Patents and Value Intellectual Property: The Uses of Patent Renewal and Application Data ». The Journal of Industrial Economics 46 (4): 405-32.

[LEN 02] LENFLE S., MIDLER C., 2002. "Stratégie d'innovation et organisation de la conception dans les entreprises amont ». Revue Française de Gestion 28 (140): 89-105.

[LER 94] LERNER J. 1994. «The Importance of Patent Scope: An Empirical Analysis ». The RAND Journal of Economics 25 (2): 319-33.

[LEY 95] LEYDESDORFF L., 1995. «The Triple Helix-University-Industry-Government Relations: A Laboratory for Knowledge-Based Economic Development ». EASST Review, 14-19.

[LOO 03] LOOY B. Van, DEBACKERE K. ANDIRES P., 2003. «Policies to Stimulate Regional Innovation Capabilities via University-industry Collaboration: An Analysis and an Assessment ». R\&D Management 33 (2): 209-29. 
[MIN 15] MINGERS, J., LEYDESDORFF L., 2015. «A review of theory and practice in scientometrics ». European Journal of Operational Research 246 (1): 1-19.

[MOW 01] MOWERY D.C., NELSON R.R., SAMPAT B.N., ZIEDONIS A.A., 2001. " The growth of patenting and licensing by U.S. universities: an assessment of the effects of the Bayh-Dole act of $1980 »$. Research Policy 30 (1): 99-119.

[NAR 85] NARIN F., NOMA E., 1985. « Is technology becoming science? » Scientometrics 7 (3-6): 369-81.

[KOR 97] NARIN F., HAMILTON K.S., OLIVASTRO D., 1997. « The increasing linkage between U.S. technology and public science ». Research Policy 26 (3): 317-30. https://doi.org/10.1016/S0048-7333(97)00013-9.

[NEL 85] NELSON R.R., WINTER S.G., 1985. An Evolutionary Theory of Economic Change. Cambridge, Mass.: Belknap Press.

[NOB 79] NOBLE D.F. America by Design: Science, Technology, and the Rise of Corporate Capitalism. Oxford University Press, Oxford, 1979.

[OST 10] Observatoire des Sciences et Techniques (OST). 2010. «Indicateurs de sciences et de technologies». Les rapports et les analyses de l'OST. Economica.

[OEC 96] OECD. 1996. « The Knowledge-based Economy - OECD ». http://www.oecd.org/sti/sci-tech/theknowledgebasedeconomy.htm.

[OEC 05] OECD. 2005. «Manuel d'Oslo: Principes directeurs pour le recueil et l'interprétation des données sur l'innovation, $3 \mathrm{e}$ édition» http://www.oecd.org/fr/science/inno/manueldosloprincipesdirecteurspourlerecueiletlinterpretationdesdonneessurlinnov ation3eedition.htm.

[SAL 01] SALTER A.J., MARTIN B.R., 2001. "The Economic Benefits of Publicly Funded Basic Research: A Critical Review." Research Policy 30 (3): 509-32.

[SAM 03] SAMPAT B.N., MOWERY D.C., ZIEDONIS A.A., 2003. "Changes in university patent quality after the Bayh-Dole act: a re-examination ». International Journal of Industrial Organization, The economics of intellectual property at universities, 21 (9): 1371-90.

[SEM 15] SEMPERE C.M., 2015. "A survey of performance issues in defence innovation». Defence and Peace Economics 28 (3): 319-43.

[SQI 13] SQUICCIARINI M., DERNIS H., CRISCUOLO C., 2013. « Measuring patent quality: indicators of technological and economic value ». OCDE.

[TEE 88] TEECE, D.J., 1988. "Capturing Value from Technological Innovation: Integration, Strategic Partnering, and Licensing Decisions.” Interfaces 18 (3): 46-61.

[TIJ 04] TIJSSEN R.J.W., 2004. « Measuring and Evaluating Science-Technology Connections and Interactions ». In Handbook of Quantitative Science and Technology Research, édité par Henk F. Moed, Wolfgang Glänzel, et Ulrich Schmoch, 695-715. Springer Netherlands.

[TRA 90] TRAJTENBERG M., 1990. «A Penny for Your Quotes: Patent Citations and the Value of Innovations ». The RAND Journal of Economics 21 (1): 172-87.

[VER 03] VERBEEK A., DEBACKERE K., LUWEL M., 2003. « Science cited in patents: A geographic "flow" analysis of bibliographic citation patterns in patents ». Scientometrics 58 (2): 241-63.

[WIP 17] WIPO. « Statistiques de propriété intellectuelle ». Consulté le 26 septembre 2017. /ipstats/fr/index.html.

\section{Annexes}

\section{Annexe 1. Les sous-classes technologiques A\&D (OST, 2010)}

B63G Offensive or defensive arrangements on vessels, mine-laying, ...

B64G Cosmonautics, vehicles or equipment therefor (apparatus for, or methods ...

C06B Explosive or thermic compositions (blasting f42d), manufacture thereof, ...

C06C Detonating or priming devices, fuses, chemical lighters, pyrophoric ...

C06D Means for generating smoke or mist, gas-attack compositions, generation ... 
C06F Matches, manufacture of matches

F41A Functional features or details common to both small arms and ordnance, ...

F41B Weapons for projecting missiles without use of explosive or combustible ...

F41C Small arms, e.g. Pistols, rifles (functional features or details common ...

$\mathrm{F} 41 \mathrm{~F} \quad$ Apparatus for launching projectiles or missiles from barrels, e.g ....

F41G Weapon sights, aiming (optical aspects thereof g02b)

F41H Armour, armoured turrets, armoured or armed vehicles, means of attack or ...

F41J Targets, target ranges, bullet catchers

F42B Explosive charges, e.g. For blasting, fireworks, ammunition (explosive ...

F42C Ammunition fuses (blasting cartridge initiators f42b0003100000, chemical ...

F42D Blasting (fuses, e.g. Fuse cords, c06c0005000000, blasting cartridges ...

Annexe 2. Distribution des familles de brevets par premier office de dépôt

Premier office de dépôt

Nombre de famille de brevets

Argentine (l')

Autriche (l')

Australie (l')

Belgique (la)

Bulgarie (la)

Brésil (le)

Canada (le)

Suisse (la)

Chili (le)

Chine (la)

Colombie (la)

Costa Rica (le)

Tchécoslovaquie

Cuba

Tchéquie (la)

Allemagne (l')

3175

Danemark (le)

Eurasian Patent Organization

European Patent Office

Espagne (1')

Finlande (la)

France (la)

Royaume-Uni de Grande-Bretagne et

d'Irlande du Nord (le)

Grèce (la)

Hong Kong

Hongrie (la)

Irlande (1')

Israël

Inde (1')

Italie (l') 
Pays-Bas (les)

Norvège (la)

Nouvelle-Zélande (la)

Pérou (le)

Philippines (les)

Pologne (la)

Portugal (le)

Roumanie (la)

Serbie (la)

Russie (la Fédération de) $\quad 57$

Arabie saoudite (l') 1

Suède (la) $\quad 720$

Singapour 26

Slovénie (la) 1

Slovaquie (la) $\quad 1$

Saint-Marin 1

Union Soviétique (USSR) $\quad 6$

Thaïlande (la) 1

Turquie (la) 11

Taïwan (Province de Chine) 120

Ukraine (1') 9

États-Unis d'Amérique (les) $\quad 38794$

Uruguay (1') 1

Venezuela (République bolivarienne du) 2

World Intellectual Property Organization
(WIPO)

Yugoslavie/Serbie et Montenegro $\quad 1$

Afrique du Sud (1') 204

Zimbabwe (le) 1

\footnotetext{
${ }^{1}$ Voir annexe 1.

${ }^{2}$ Orbit-Questel est une banque de données spécialisée dans la propriété intellectuelle, et en particulier sur les brevets.

${ }^{3}$ L'EPO définit une famille de brevets comme comprenant tous les documents ayant exactement la même priorité ou la même combinaison de priorités.
} 